\title{
Theory of the $\beta$-type Organic Superconductivity under Uniaxial Compression
}

\author{
Takeo Suzuki, Seiichiro Onari, Hiroshi Ito, and Yukio Tanaka \\ Department of Applied Physics, Nagoya University, Chikusa, Nagoya 464-8603, Japan
}

We study theoretically the shift of the superconducting transition temperature $\left(T_{c}\right)$ under uniaxial compression in $\beta$-type organic superconductors, $\beta$-(BEDT-TTF $)_{2} \mathrm{I}_{3}$ and $\beta$-(BDA-TTP $)_{2} \mathrm{X}\left[\mathrm{X}=\mathrm{SbF}_{6}, \mathrm{AsF}_{6}\right]$, in order to clarify the electron correlation, the spin frustration, and the effect of dimerization. The transfer integrals are calculated by the extended Hückel method assuming the uniaxial strain, and the superconducting state mediated by the spin fluctuation is solved using Eliashberg's equation with the fluctuation-exchange approximation. The calculation is carried out on both the dimerized (one-band) and nondimerized (two-band) Hubbard models. We have found that (i) the behavior of $T_{c}$ in $\beta$-(BEDT-TTF $)_{2} \mathrm{I}_{3}$ with a stronger dimerization is well reproduced by the dimer model, while that in weakly dimerized $\beta$-BDA-TTP salts is rather well reproduced by the two-band model, and (ii) the competition between the spin frustration and the effect induced by the fluctuation is important in these materials, which causes the nonmonotonic shift of $T_{c}$ against uniaxial compression.

KEYWORDS: superconductivity, organic superconductor, uniaxial compression, spin frustration, spin fluctuation

\section{Introduction}

The superconductivity of the $\kappa$-type BEDT-TTF salts has fascinated experimental and theoretical researchers with a high transition temperature $T_{c}$ over $10 \mathrm{~K} .{ }^{1)}$ In the $\kappa$-type BEDT-TTF salts, donors are considered to form dimers. ${ }^{2)}$ Then, the split antibonding HOMO band is half-filled with the effective on-site Coulomb interaction $(U)$ close to the bandwidth. It is considered to be a strongly correlated electron system, where the superconductivity is mediated by the antiferromagnetic spin fluctuation. ${ }^{3-7)}$ The spin fluctuation is suppressed by the geometrical spin frustration since the donors locate on an anisotropic triangular lattice. The effect becomes strongest on a regular triangular lattice, which is considered to be realized in $\kappa$-(BEDT-TTF $\left.)_{2} \mathrm{Cu}_{2}(\mathrm{CN})_{3} \cdot{ }^{8,9}\right)$ The organic superconductors with $\beta$-type donor arrangement also exhibit a relatively 
high $T_{c}$ reaching $8 \mathrm{~K}$. As shown in Fig. 1, donors stack unidirectionally and dimerize on a triangular lattice. However, the dimerization of the molecules is weaker than that of the $\kappa$-type salts. Then, as an effective model for the $\beta$-type salt, not only the dimer model but also the nondimerized original two-band model should be taken into account.

Uniaxial compression is a powerful technique for studying the strongly correlated electron systems since transfer integrals can be controlled selectively. ${ }^{10)}$ The spin frustration and the electron correlation are controlled by uniaxial compression. In fact, the shift of $T_{c}$ under the uniaxial compression in $\kappa$-(BEDT-TTF $)_{2} \mathrm{Cu}(\mathrm{NCS})_{2}$ can be explained by the term of the spin frustration on an anisotropic triangular lattice composed of dimerized molecules. ${ }^{11)}$ Our group reported the shift of $T_{c}$ in $\beta$-type salts, $\beta$-(BDA-TTP $)_{2} \mathrm{X}\left[\mathrm{X}=\mathrm{SbF}_{6}, \mathrm{AsF}_{6}\right]$, under uniaxial compression parallel and perpendicular to the molecular stack direction. ${ }^{12)}$ Under the compression perpendicular to the stack, $T_{c}$ shows a nonmonotonic behavior, once increases and reaches a maximum and decreases subsequently, as a function of the applied piston pressure. On the basis of the dimer model of the anisotropic triangular lattice, this behavior has been attributed to the result of the competition of the enhancements of the spin fluctuation and the spin frustration effect. However, the data for $\beta$-(BDA-TTP $)_{2} \mathrm{AsF}_{6}$ was not well reproduced. Furthermore, for $\beta$-(BEDT-TTF $)_{2} I_{3}, T_{c}$ exhibits nonmonotonic behaviors for both compression directions, in strong contrast to that of the BDA-TTP salts. ${ }^{13)}$

In order to clarify the behavior of $T_{c}$ under uniaxial compression and obtain insight into the superconductivity of $\beta$-type salts, we study theoretically the superconductivity mediated by the spin fluctuation on both the original two-band Hubbard model and the dimerized Hubbard model using the fluctuation exchange (FLEX) approximation ${ }^{14,15)}$ for $\beta$-(BEDT-TTF $)_{2} \mathrm{I}_{3}$ and $\beta$-(BDA-TTP $)_{2} \mathrm{X}\left[\mathrm{X}=\mathrm{SbF}_{6}, \mathrm{AsF}_{6}\right]$. Measurements of STM and specific heat are consistent with the $d$-wave symmetry of BDA-TTP salts. ${ }^{16,17)}$ It is noted that the zero bias conductance peak due to the Andreev bound state ${ }^{18,19)}$ has been reported very recently. ${ }^{20)}$ Thus, it is natural to employ the spin fluctuation theory, which can realize a $d$-wave gap function beyond the BCS theory.

Although some of the results have been reported in our previous papers, ${ }^{12,13,21)}$ in this paper, we note the difference in the strength of the dimerization among the three materials, which is evaluated by the ratio of the transfer integrals along the stacking direction, namely, $t_{p 1}$ and $t_{p 2}$, as shown in Fig. 1 . We have found that the dimer model is suitable to the strongly dimerized $\beta$-(BEDT-TTF $)_{2} \mathrm{I}_{3}$ among the three materials, $t_{p 1} / t_{p 2}=2.99$, while the original two-band model is suitable for the weakly dimerized 
$\beta$ - $(\mathrm{BDA}-\mathrm{TTP})_{2} \mathrm{AsF}_{6}$ among the three materials, $t_{p 1} / t_{p 2}=2.02$. We have also found that the nonmonotonic behavior of $T_{c}$ is due to the competition between the spin frustration and the effect of the self-energy induced by the spin fluctuation.

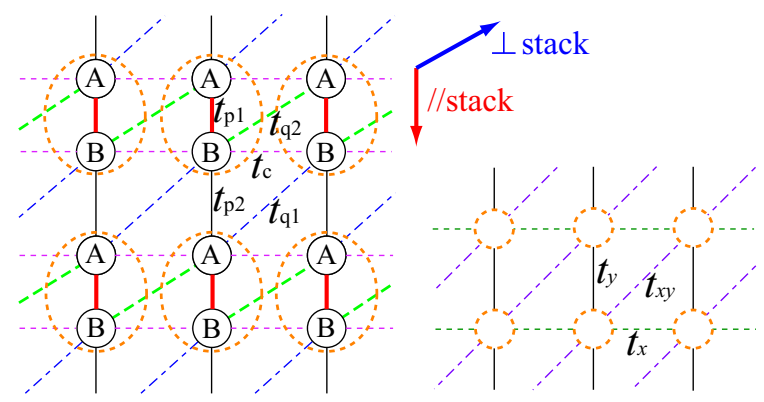

Fig. 1. (Color online) Lattice structure on a two-band model (left) and the dimer model (right) for $\beta$-type salts. Donor molecules locate at A and B sites, and form dimers, as depicted by the dotted ellipsoids.

\section{Formulation}

In the following, we introduce the models that we employ in this study. Putting a donor molecule into a site on a lattice, the crystal structure of $\beta$-type salts is modeled by a triangular lattice with A and B sublattices, as shown in Fig. 1. Here, we distort the lattice in order to make the reciprocal lattice rectangular. Then, the direction perpendicular to the donor stack direction is distorted, as illustrated in Fig. 1. However, this distortion makes no changes in physical quantities, such as $T_{c}$, because the topology of the lattice is invariant. According to the dimer model, the sublattices are dimerized, and the original two-band model is reduced to the one-band model (dimer model), as shown in Fig. 1. In the dimer model, hopping integrals are given by $t_{x}=-t_{\mathrm{q} 2} / 2+t_{c}$, $t_{x y}=-t_{\mathrm{q} 1} / 2$, and $t_{y}=-t_{\mathrm{p} 2} / 2$ using the hopping integrals in the original two-band model. ${ }^{22}$ Hopping integrals under uniaxial compression are also estimated by the extended Hückel method ${ }^{23)}$ assuming uniform displacements of molecules from the structure at ambient pressure. $\left.{ }^{12}\right)$ The validity of the Huckel method as a first approximation is implied because it can predict the round Fermi surface, which is consistent with the Shubnikov-de Haas (SdH) or de Haas-van Alphen (dHvA) measurements. ${ }^{24,25)}$ The uniaxial strain under uniaxial compression is assumed to be $0.3 \% / \mathrm{kbar}$ according to the results of X-ray measurements. ${ }^{26)}$ We also assume that the encapsulation of the sample with epoxy, which is prepared for uniaxial compression, induces an isotropic 
Table I. Transfer integrals under uniaxial compression in the $\beta$-type salts, where unit of transfer integrals is $10^{-2} \mathrm{eV}$.

\begin{tabular}{|c|c|c|c|c|c|c|}
\hline & Pressure [kbar] & $t_{c}$ & $t_{p 1}$ & $t_{p 2}$ & $t_{q 1}$ & $t_{q 2}$ \\
\hline$\beta$-(BEDT-TTF $)_{2} \mathrm{I}_{3}$ & 0 & -5.22 & 24.5 & 8.23 & 12.6 & 6.91 \\
\cline { 2 - 7 } & $/ /$ stack 5 & -4.75 & 26.8 & 9.16 & 13.0 & 7.07 \\
\cline { 2 - 7 } & $\perp$ stack 5 & -5.98 & 24.0 & 8.15 & 13.1 & 7.25 \\
\hline$\beta$-(BDA-TTP) ${ }_{2} \mathrm{SbF}_{6}$ & 0 & -0.45 & 15.0 & 6.43 & 8.34 & 9.04 \\
\cline { 2 - 7 } & $/ /$ stack 5 & -0.02 & 16.3 & 7.12 & 8.58 & 9.23 \\
\cline { 2 - 7 } & $\perp$ stack 5 & -0.85 & 14.8 & 6.38 & 8.68 & 9.60 \\
\hline$\beta$-(BDA-TTP $)_{2} \mathrm{AsF}_{6}$ & 0 & -0.36 & 15.5 & 7.74 & 8.52 & 9.79 \\
\cline { 2 - 7 } & $/ /$ stack 5 & 0.07 & 16.8 & 8.45 & 8.72 & 9.99 \\
\cline { 2 - 7 } & $\perp$ stack 5 & -0.78 & 15.2 & 7.69 & 8.83 & 10.5 \\
\hline
\end{tabular}

pressure of $1 \mathrm{kbar}$ in the sample. We show the obtained transfer integrals under uniaxial compression in $\beta$-type salts in Table $\mathrm{I}$.

In this paper, we employ both the two-band and one-band Hubbard models with the repulsion $U$ on a triangular lattice,

$$
\begin{aligned}
\mathcal{H}= & \sum_{\langle i, j\rangle, \sigma} \sum_{\alpha, \beta}^{\mathrm{A}, \mathrm{B}} t_{i j}\left(c_{i \sigma}^{\alpha \dagger} c_{j \sigma}^{\beta}+\text { H.c. }\right) \\
& +U \sum_{i} \sum_{\alpha}^{\mathrm{A}, \mathrm{B}} n_{i \uparrow}^{\alpha} n_{i \downarrow}^{\alpha},
\end{aligned}
$$

in standard notations and calculate $T_{c}$ by solving Eliashberg's equation within the FLEX approximation. ${ }^{27,28)}$ In the two-band model, Green's function $G$, the self-energy $\Sigma$, the susceptibility $\chi$, and the gap function $\phi$ are all $2 \times 2$ matrices, such as $G_{\alpha \beta}(k)$, where $\alpha, \beta=\mathrm{A}, \mathrm{B}$ and $k \equiv\left(\boldsymbol{k}, i \omega_{n}\right)$ with $\omega_{n}=(2 n-1) \pi T$ being the Matsubara frequency for fermions.

The self-energy is given by

$$
\Sigma_{\alpha \beta}(k)=\frac{T}{N} \sum_{q} G_{\alpha \beta}(k-q) V_{\alpha \beta}^{(1)}(q),
$$

where the FLEX effective interaction $V(q)$ for the self-energy is

$$
\begin{aligned}
V_{\alpha \beta}(q)= & \frac{3}{2} U^{2}\left[\frac{\chi^{\operatorname{irr}}(q)}{1-U \chi^{\operatorname{irr}}(q)}\right]_{\alpha \beta}+\frac{1}{2} U^{2}\left[\frac{\chi^{\operatorname{irr}}(q)}{1+U \chi^{\operatorname{irr}}(q)}\right]_{\alpha \beta} \\
& -U^{2} \chi_{\alpha \beta}^{\operatorname{irr}}(q)
\end{aligned}
$$


with the irreducible susceptibility

$$
\chi_{\alpha \beta}^{\mathrm{irr}}(q)=-\frac{T}{N} \sum_{k} G_{\alpha \beta}(k+q) G_{\beta \alpha}(k) .
$$

Here, we denote $q \equiv\left(\boldsymbol{q}, i \epsilon_{l}\right)$ with $\epsilon_{l}=2 \pi l T$ being the Matsubara frequency for bosons, and $N$ as the number of $\boldsymbol{k}$-points on a mesh.

With Dyson's equation,

$$
\left[G(k)^{-1}\right]_{\alpha \beta}=\left[G^{0}(k)^{-1}\right]_{\alpha \beta}-\Sigma_{\alpha \beta}(k),
$$

where $G^{0}$ is the bare Green's function $G_{\alpha \beta}^{0}(k)=\left[\left(i \omega_{n}+\mu-\varepsilon_{\boldsymbol{k}}^{0}\right)^{-1}\right]_{\alpha \beta}$ with $\varepsilon_{\boldsymbol{k}}^{0}$ being the bare energy, we have solved eqs. (2)-(5) self-consistently to obtain Green's functions under FLEX interactions.

Then, $T_{c}$ is obtained from Eliashberg's equation for the spin-singlet pairing,

$$
\begin{aligned}
\lambda \phi_{\alpha \beta}(k)= & -\frac{T}{N} \sum_{k^{\prime}} \sum_{\alpha^{\prime}, \beta^{\prime}}^{\mathrm{A}, \mathrm{B}} V_{\alpha \beta}^{\sin }\left(k-k^{\prime}\right) \\
& \times G_{\alpha \alpha^{\prime}}\left(k^{\prime}\right) G_{\beta \beta^{\prime}}\left(-k^{\prime}\right) \phi_{\alpha^{\prime} \beta^{\prime}}\left(k^{\prime}\right),
\end{aligned}
$$

where $\phi$ is the gap function, and the spin singlet pairing interaction $V^{\sin }(q)$ is given as

$$
\begin{aligned}
V_{\alpha \beta}^{\sin }(q)= & \frac{3}{2} U^{2}\left[\frac{\chi^{\operatorname{irr}}(q)}{1-U \chi^{\operatorname{irr}}(q)}\right]_{\alpha \beta}-\frac{1}{2} U^{2}\left[\frac{\chi^{\operatorname{irr}}(q)}{1+U \chi^{\operatorname{irr}}(q)}\right]_{\alpha \beta} \\
& +U \delta_{\alpha \beta} .
\end{aligned}
$$

From eq. (6), $T_{c}$ is determined as the temperature at which the maximum eigenvalue $\lambda$ becomes unity. However, because the performance of the computer is not sufficient for deducing $T_{c}$ directly, we evaluate $\lambda$ at a constant temperature of $T / t_{q 1}=0.01$ as a function of the piston pressure for the uniaxial compression. The larger value of $\lambda$ corresponds to the higher $T_{c}$.

At this temperature, the $\boldsymbol{k}$-dependence of the spin susceptibility $\chi^{s}$ is expressed by the diagonalized component

$$
\chi_{\mathrm{s}}=\frac{\chi_{\mathrm{AA}}^{\mathrm{s}}+\chi_{\mathrm{BB}}^{\mathrm{s}}}{2}+\sqrt{\left[\frac{\chi_{\mathrm{AA}}^{\mathrm{s}}-\chi_{\mathrm{BB}}^{\mathrm{s}}}{2}\right]^{2}+\left|\chi_{\mathrm{AB}}^{\mathrm{s}}\right|^{2}},
$$

where the matrix of the spin susceptibility is

$$
\chi_{\alpha \beta}^{\mathrm{s}}(\boldsymbol{k}, 0)=\left[\frac{\chi^{\operatorname{irr}}(\boldsymbol{k}, 0)}{1-U \chi^{\operatorname{irr}}(\boldsymbol{k}, 0)}\right]_{\alpha \beta} .
$$

Throughout this study, we consider $t_{\mathrm{q} 1}$ under ambient pressure as a unit of energy, $U=$ 10 on the two-band model and $U=2 t_{\mathrm{p} 1}$ on the dimer model. The system with $U=10$ corresponds to the strongly correlated electron system. In the numerical calculation, we 


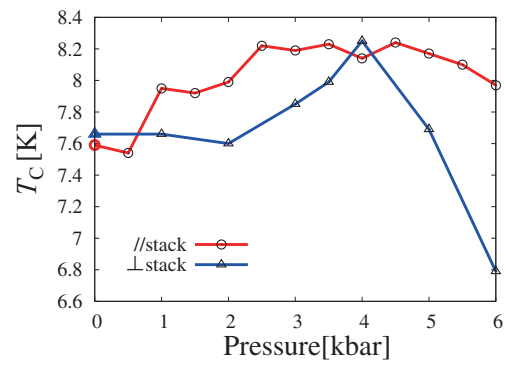

Fig. 2. (Color online) Experimental data of $T_{c}$ under uniaxial compression in $\beta$-(BEDT-TTF $)_{2} \mathrm{I}_{3}$ defined by the midpoint of the transition. ${ }^{13)}$

consider $N=128 \times 128 \boldsymbol{k}$-point meshes and the Matsubara frequencies from $\epsilon_{n}$ from $-\left(2 N_{c}-1\right) \pi T$ to $\left(2 N_{c}-1\right) \pi T$ with $N_{c}=4096$.

\section{Result}

\section{$3.1 \beta-(B E D T-T T F)_{2} I_{3}$}

We begin with $\beta$-(BEDT-TTF $)_{2} \mathrm{I}_{3}$ in which the dimerization is the strongest among the three salts. Although it is known that the $T_{c}$ of $\beta$-(BEDT-TTF $)_{2} \mathrm{I}_{3}$ jumps from $1.5 \mathrm{~K}$ at ambient pressure up to $8 \mathrm{~K}$ at a pressure of $1 \mathrm{kbar},{ }^{29)}$ only the $8 \mathrm{~K}$ superconductivity phase is observed under epoxy encapsulation because of the slight pressure induced by the epoxy. ${ }^{13)}$ Figure 2 shows that the experimental $T_{c}$ increases with uniaxial compression along both the $/ /$ stack and $\perp$ stack directions up to $4 \mathrm{kbar}$, and decreases in further piston pressures. ${ }^{13)}$ It is very interesting that $T_{c}$ shows a nonmonotonic behavior against the uniaxial compression.
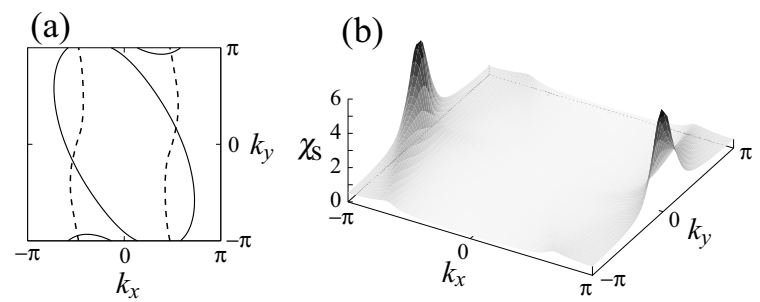

Fig. 3. (a) Fermi surface (solid line) and nodes of gap function (dotted line) and (b) $\chi_{s}$ obtained using the two-band model for $\beta$-(BEDT-TTF $)_{2} \mathrm{I}_{3}$ at ambient pressure.

We first examine the gap function and the Fermi surface. The gap function obtained using eq. (6) is a $d$-wave that changes sign four times on the Fermi surface, as shown in Fig. 3. The Fermi surface obtained using the two-band model is closed owing to the 
periodicity of the Brillouin zone (BZ), which is consistent with the previous result ${ }^{30}$ ) and dHvA measurements. ${ }^{24,25)}$ The Fermi surface in the two-band model is almost the same as that in the dimer model. The peak of $\chi_{\mathrm{s}}$ is observed at the wave number $(\pi, 0)$. The gap function changes sign along the nesting vector $\boldsymbol{Q}=(\pi, 0)$. We consider that $\beta$-(BEDT-TTF $)_{2} \mathrm{I}_{3}$ is close to the SDW phase because the peak value of $\chi_{\mathrm{s}}$ is large $(\sim 6)$.

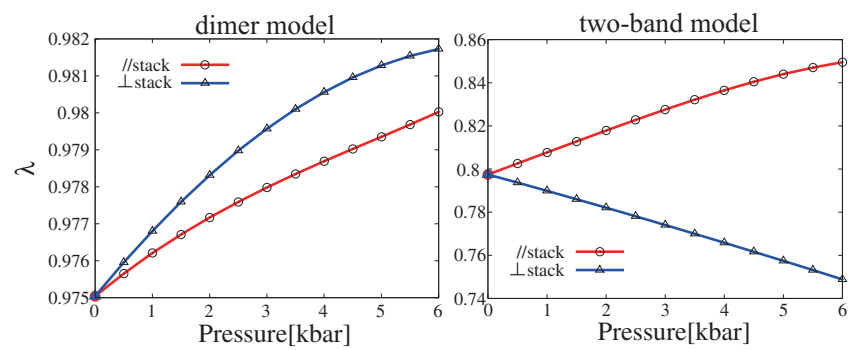

Fig. 4. (Color online) $\lambda$ under uniaxial compression on the dimer model (left panel) and two-band model (right panel) for $\beta$-(BEDT-TTF) ${ }_{2} \mathrm{I}_{3}$.

Next, we see the shift of $\lambda$, i.e., $T_{c}$ under uniaxial compression. Assuming a linear response to the uniaxial strain, the experimental results show an increase in $T_{c}$ under low piston pressures along both directions. Here, the absolute value of $\lambda$ is not important. Thus, we focus on the shift of $\lambda$ around ambient pressure. In the case of the dimer model, $\lambda$ increases with uniaxial compression along both the //stack and $\perp$ stack directions at low pressures, as shown in Fig. 4. The trend of the increasing $\lambda$ along both compression directions agrees with the experimental result at low pressures, although the larger $\lambda$ under compression perpendicular to the stacks does not match with the order of the experimental $T_{c}$.

On the other hand, in the case of the two-band model, $\lambda$ increases with uniaxial compression along the $/ /$ stack direction, while $\lambda$ decreases along the $\perp$ stack direction. This result does not match the experimental results. Thus, the dimer model is more suitable for $\beta$-(BEDT-TTF $)_{2} \mathrm{I}_{3}$, which reproduces the shift of $T_{c}$ at low pressures, than the two-band model. However, the nonmonotonic behavior of $T_{c}$, i.e., the subsequent decrease in $T_{c}$ at high pressures, can be reproduced by neither model. 


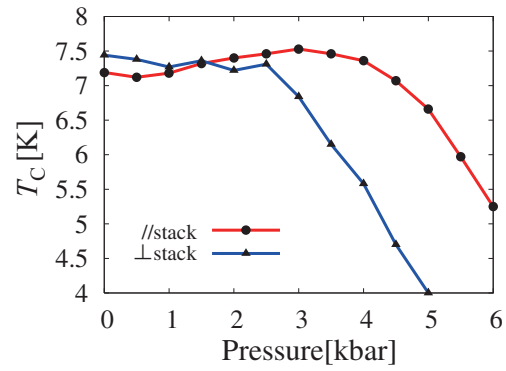

Fig. 5. (Color online) Experimental data of $T_{c}$ under uniaxial compression in $\beta-(\mathrm{BDA}-\mathrm{TTP})_{2} \mathrm{SbF}{ }_{6}$ defined by the midpoint of the transition. ${ }^{12)}$

\section{2 $\beta-(B D A-T T P)_{2} S b F_{6}$}

Next, we move on to $\beta$-(BDA-TTP $)_{2} \mathrm{SbF}_{6}$. Under uniaxial compression along the //stack direction, experimental data of $T_{c}$ increases with pressure, reaching a maximum at 3kbar, and decreases in further pressures, as shown in Fig. 5. On the other hand, along the $\perp$ stack direction, $T_{c}$ decreases monotonically with pressure. $\left.{ }^{12}\right)$
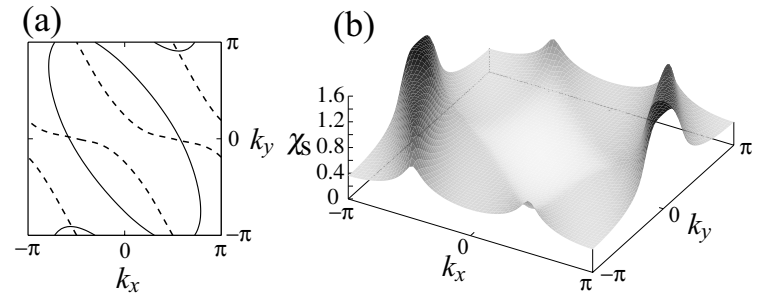

Fig. 6. (a) Fermi surface (solid line) and nodes of gap function (dotted line) and (b) $\chi_{s}$ obtained using the two-band model for $\beta$-(BDA-TTP $)_{2} \mathrm{SbF}_{6}$ at ambient pressure.

The gap function is also calculated to be a $d$-wave, as shown in Fig. 6(a). The obtained Fermi surface and symmetry of the gap function are consistent with previous calculations $^{31,32)}$ and STM measurements, ${ }^{16)}$ indicating the four-fold symmetry of the gap function. The $d$-wave is mediated by the spin fluctuation induced by the nesting vector. Thus, it is also reasonable to apply the spin fluctuation theory to this system. As shown in Fig. 6(b), the peak of $\chi_{\mathrm{s}}$ is found at the wave number $\left(\frac{9}{10} \pi, \frac{3}{4} \pi\right)$; however, the peak value of $\chi_{\mathrm{s}}$ is small $(\sim 1.4)$.

Then, we observe the shift of $\lambda$ under uniaxial compression in Fig. 7. In the case of the dimer model, $\lambda$ increases with pressure reaching a maximum at $2 \mathrm{kbar}$, and decreases in further pressures under uniaxial compression along the //stack direction, while along the $\perp$ stack direction, $\lambda$ decreases monotonically with increasing pressure. This behavior 


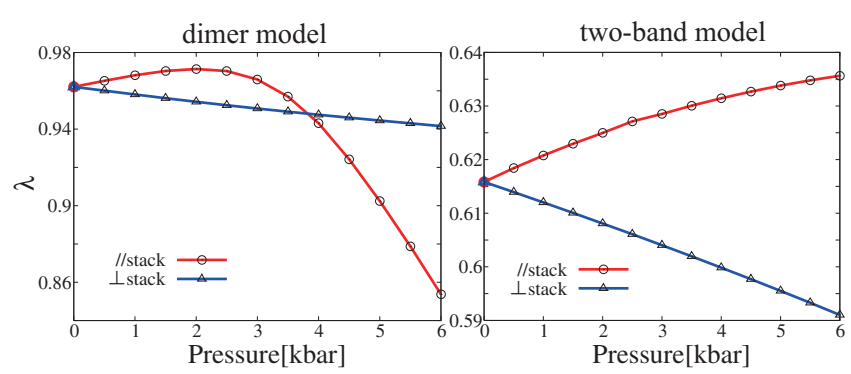

Fig. 7. (Color online) $\lambda$ under uniaxial compression on the dimer model (left panel) and the twoband model (right panel) for $\beta$-(BDA-TTP) ${ }_{2} \mathrm{SbF}_{6}$.

is consistent with the experimental results. On the other hand, in the case of the twoband model, $\lambda$ increases monotonically with pressure under uniaxial compression along the //stack direction, while $\lambda$ decreases monotonically under uniaxial compression along the $\perp$ stack direction.

In $\beta$-(BDA-TTP $)_{2} \mathrm{SbF}_{6}$, a maximum $\lambda$ value is obtained on the dimer model, which is not obtained in $\beta$-(BEDT-TTF $)_{2} \mathrm{I}_{3}$. In the following, we discuss the origin of the nonmonotonic behavior of $\lambda$ under uniaxial compression calculated in the dimer model.

First, we focus on $\left\langle U^{2} \chi_{\mathrm{S}}\right\rangle_{\mathrm{B}}$ that is the mean value of $U^{2} \chi_{\mathrm{s}}$ within the BZ, which is a significant term in Eliashberg's equation (6). In the case of the dimer model, the on-site Coulomb interaction $U=2 t_{\mathrm{p} 1}$ depends on the uniaxial compression. Thus, the value of $U^{2}$ in the effective interaction is also important when we discuss the uniaxial compression dependence of $\lambda$. As shown in Fig. 8, under uniaxial compression along

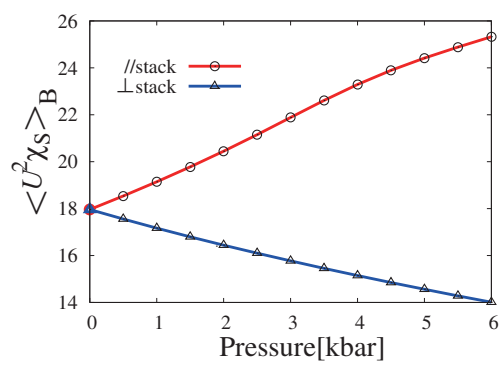

Fig. 8. (Color online) $\left\langle U^{2} \chi_{\mathrm{S}}\right\rangle_{\mathrm{B}}$ under uniaxial compression on the dimer model for $\beta$-(BDA$\mathrm{TTP})_{2} \mathrm{SbF}_{6}$.

the //stack direction, $\left\langle U^{2} \chi_{\mathrm{s}}\right\rangle_{\mathrm{B}}$ increases monotonically with pressure, while $\left\langle U^{2} \chi_{\mathrm{s}}\right\rangle_{\mathrm{B}}$ decreases under uniaxial compression along the $\perp$ stack direction. Here, we note the geometrical spin frustration induced by the triangular lattice. We denote $t^{\prime}\left(=t_{y}\right)$ as the 
hopping integral along the dimerized direction and $t\left(=\left(t_{x}+t_{x y}\right) / 2\right)$ as the mean value of other hopping integrals in the dimer model. In this material, $t^{\prime} / t<1$ is satisfied at ambient pressure, and then the geometrical spin frustration becomes strong under uniaxial compression along the //stack direction, while it becomes weak under uniaxial compression along the $\perp$ stack direction, which is consistent with the results of $\left\langle U^{2} \chi_{\mathrm{s}}\right\rangle_{\mathrm{B}}$.

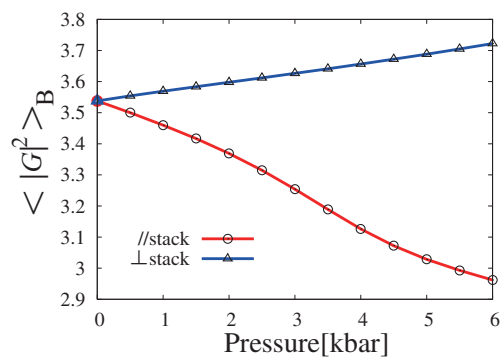

Fig. 9. (Color online) $\left\langle|G|^{2}\right\rangle_{\mathrm{B}}$ under uniaxial compression on the dimer model for $\beta$-(BDA$\mathrm{TTP})_{2} \mathrm{SbF}_{6}$.

Next, we show in Fig. 9 the mean value of $|G|^{2}$ in the BZ, which is another important term in eq. (6). Under uniaxial compression along the //stack direction, the value of $\left\langle|G|^{2}\right\rangle_{\mathrm{B}}$ decreases monotonically with increasing pressure, while that increases monotonically along the $\perp$ stack direction. Under uniaxial compression along the //stack direction, the increase in $\left\langle U^{2} \chi_{\mathrm{s}}\right\rangle_{\mathrm{B}}$ strongly competes with the decrease in $\left\langle|G|^{2}\right\rangle_{\mathrm{B}}$. Thus, the nonmonotonic behavior reaching a maximum at the value of $\lambda$ is considered to be caused by such competition.

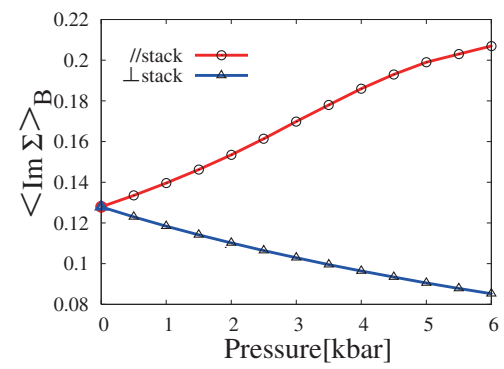

Fig. 10. (Color online) $\langle|\operatorname{Im} \Sigma|\rangle_{\mathrm{B}}$ under uniaxial compression on the dimer model for $\beta$-(BDA$\mathrm{TTP})_{2} \mathrm{SbF}_{6}$.

Now, we consider the origin of the uniaxial compression dependence in $\left\langle|G|^{2}\right\rangle_{\mathrm{B}}$. $G$ is suppressed by the imaginary part of self-energy $\operatorname{Im} \Sigma$. In Fig. 10, we show the com- 
pression dependence of $\langle|\operatorname{Im} \Sigma|\rangle_{\mathrm{B}}$, which is the mean value of $|\operatorname{Im} \Sigma|$ in the BZ. Under uniaxial compression along the //stack direction, the value of $\langle|\operatorname{Im} \Sigma|\rangle_{\mathrm{B}}$ increases monotonically with pressure, while it decreases monotonically along the $\perp$ stack direction. These results are consistent with the results of $\left\langle|G|^{2}\right\rangle_{\mathrm{B}}$. Thus, under uniaxial compression along the $/ /$ stack direction, the increase in the value of $\langle|\operatorname{Im} \Sigma|\rangle_{\mathrm{B}}$ reduces the value of $\left\langle|G|^{2}\right\rangle_{\mathrm{B}}$, which competes with the increase in the value of $\left\langle U^{2} \chi_{\mathrm{s}}\right\rangle_{\mathrm{B}}$

Compared with the experimental results, the low-pressure behavior of $T_{c}$ can be reproduced by both the dimer and two-band models. We observe that the dimer model is more suitable than the two-band model, because the maximum of $T_{c}$ can be reproduced by the dimer model.

\section{$3.3 \beta-(B D A-T T P)_{2} A s F_{6}$}

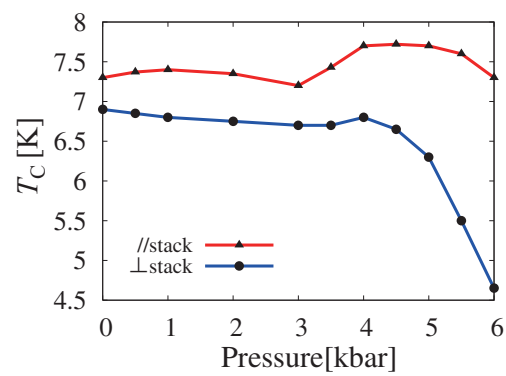

Fig. 11. (Color online) Experimental data of $T_{c}$ under uniaxial compression in $\beta$-(BDA-TTP $)_{2} \mathrm{AsF}_{6}$ defined by the midpoint of the transition. ${ }^{12)}$

Finally, we move on to $\beta$-(BDA-TTP $)_{2} \mathrm{AsF}_{6}$. As shown in Fig. 11, under uniaxial compression along the //stack direction, the experimental data of $T_{c}$ is almost constant up to $3 \mathrm{kbar}$ and increases with pressure, reaching a maximum at $4.5 \mathrm{kbar}$, and decreases in further pressures. On the other hand, along $\perp$ stack direction, $T_{c}$ decreases monotonically with increasing pressure. ${ }^{12)}$

As shown in Fig. 12, the calculated gap function is also a $d$-wave, and the shape of the Fermi surface is consistent with previous results ${ }^{31)}$ and STM measurements. ${ }^{16)}$ The peak of $\chi_{\mathrm{s}}$ is found at the wave number $\left(\pi, \frac{2}{5} \pi\right)$, but the peak value of $\chi_{\mathrm{s}}$ is small $(\sim 1.2)$. We can assume that this material is far from the SDW phase owing to the small value of $\chi_{\mathrm{s}}$.

Figure 13 shows that in the dimer model, $\lambda$ increases only very slightly up to 1 kbar and decreases monotonically with increasing pressure under uniaxial compression 
(a)

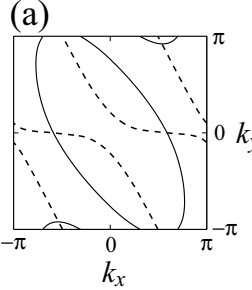

(b)

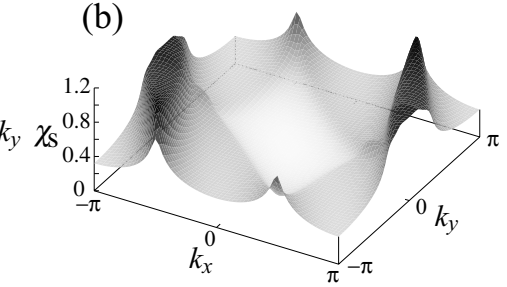

Fig. 12. (a) Fermi surface (solid line) and nodes of gap function (dotted line) and (b) $\chi_{s}$ obtained using the two-band model in $\beta$-(BDA-TTP $)_{2} \mathrm{AsF}_{6}$ at ambient pressure.

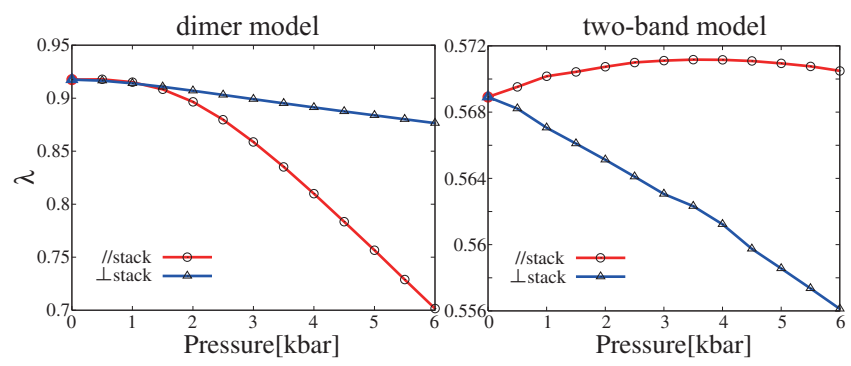

Fig. 13. (Color online) $\lambda$ under uniaxial compression on the dimer model (left panel) and two-band model (right panel) for $\beta$-(BDA-TTP) ${ }_{2} \mathrm{AsF}_{6}$.

along the $/ /$ stack direction and $\lambda$ also decreases monotonically along $\perp$ stack direction, which does not fit with the experimental results. ${ }^{12)}$ On the other hand, in the twoband model, $\lambda$ increases with pressure, reaching a maximum at 3.5kbar, and decreases in further pressures under uniaxial compression along the //stack direction, while $\lambda$

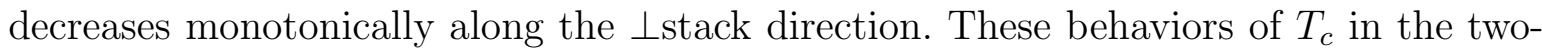
band model are consistent with those observed by experiment. Thus, the two-band model is more suitable for reproducing the behavior of $T_{c}$ in $\beta$-(BDA-TTP) ${ }_{2} \mathrm{AsF}_{6}$.

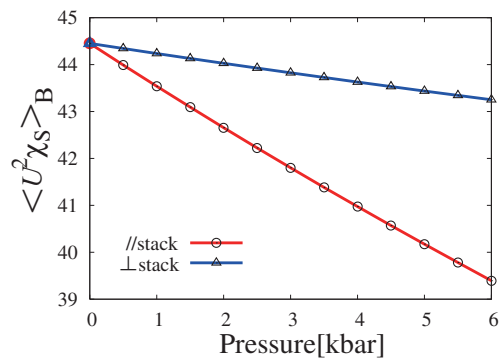

Fig. 14. (Color online) $\left\langle U^{2} \chi_{\mathrm{S}}\right\rangle_{\mathrm{B}}$ under uniaxial compression on the two-band model for $\beta$-(BDA$\mathrm{TTP})_{2} \mathrm{AsF}_{6}$. 


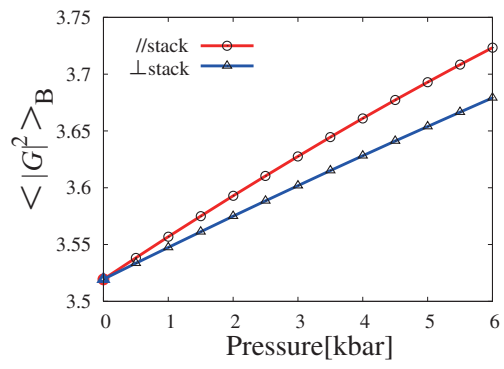

Fig. 15. (Color online) $\left\langle|G|^{2}\right\rangle_{\mathrm{B}}$ under uniaxial compression on the two-band model for $\beta$-(BDA$\mathrm{TTP})_{2} \mathrm{AsF}_{6}$.

Hereafter, we discuss the origin of the nonmonotonic behavior of $\lambda$ in the two-band model. As shown in Fig. 14, in the two-band model, $\left\langle U^{2} \chi_{\mathrm{S}}\right\rangle_{\mathrm{B}}$ decreases with increasing pressure under uniaxial compression along both the //stack and Lstack directions. In Fig. 15, we show the result of $\left\langle|G|^{2}\right\rangle_{\mathrm{B}}$ that is the mean value of the largest eigenvalue of $|G|^{2}$ in the BZ. Under uniaxial compression along both the //stack and $\perp$ stack directions, the value of $\left\langle|G|^{2}\right\rangle_{\mathrm{B}}$ increases monotonically with pressure. Thus, under uniaxial compression along the $/ /$ stack direction, the decrease in $\left\langle U^{2} \chi_{\mathrm{s}}\right\rangle_{\mathrm{B}}$ and the increase in $\left\langle|G|^{2}\right\rangle_{\mathrm{B}}$ strongly compete, as in the case of $\beta$-(BDA-TTP $)_{2} \mathrm{SbF}_{6}$. The strong competition between $\left\langle U^{2} \chi_{\mathrm{S}}\right\rangle_{\mathrm{B}}$ and $\left\langle|G|^{2}\right\rangle_{\mathrm{B}}$ is the origin of the nonmonotonic behavior of the value of $\lambda$.

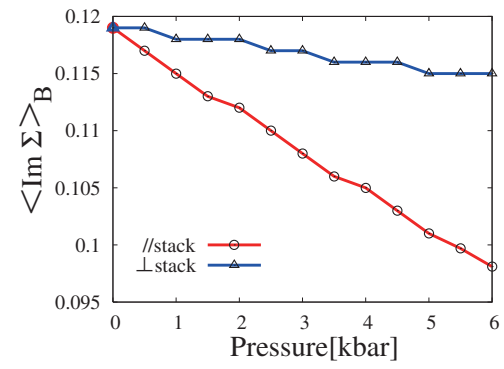

Fig. 16. $\langle\operatorname{Im} \Sigma\rangle_{\mathrm{B}}$ under uniaxial compression on the two-band model for $\beta$-(BDA-TTP $)_{2} \mathrm{AsF}_{6}$.

The uniaxial compression dependence of $\left\langle|G|^{2}\right\rangle_{\mathrm{B}}$ is explained by the largest eigenvalue of $\langle|\operatorname{Im} \Sigma|\rangle_{\mathrm{B}}$ as shown in Fig. 16, as in the case of $\beta$-(BDA-TTP $)_{2} \mathrm{SbF}_{6}$. Under uniaxial compression along the both $/ /$ stack and $\perp$ stack directions, the value of $\langle|\operatorname{Im} \Sigma|\rangle_{\mathrm{B}}$ decreases monotonically with increasing pressure. In particular, along the $/ /$ stack direction, the value of $\langle|\operatorname{Im} \Sigma|\rangle_{\mathrm{B}}$ decreases rapidly. This decrease in the value of $\langle|\operatorname{Im} \Sigma|\rangle_{\mathrm{B}}$ increases the value of $\left\langle|G|^{2}\right\rangle_{\mathrm{B}}$. 
Table II. Relationship between the dimerization factor $\left(t_{\mathrm{p} 1} / t_{\mathrm{p} 2}\right)$ and the suitable model for the three $\beta$-type salts.

\begin{tabular}{|c|c|c|c|}
\hline & $\beta$-(BEDT-TTF $)_{2} \mathrm{I}_{3}$ & $\beta$-(BDA-TTP $)_{2} \mathrm{SbF}_{6}$ & $\beta$-(BDA-TTP $)_{2} \mathrm{AsF}_{6}$ \\
\hline dimerization factor $\left(t_{\mathrm{p} 1} / t_{\mathrm{p} 2}\right)$ & 2.98 & 2.30 & 2.00 \\
\hline dimer model & $\bigcirc$ & $\bigcirc$ & $\times$ \\
\hline 2 band model & $\times$ & $\bigcirc$ & $\bigcirc$ \\
\hline
\end{tabular}

\section{Discussion}

As a summary of the results, we show Table II that indicates the suitable model for explaining the experimental results of $T_{c}$, mainly focusing on the reproducibility of the experimental $T_{c}$ in a low pressure range, where the linearity of the uniform strain seems to be a good approximation. The strength of dimerization is estimated by the dimerization factor $\left(t_{\mathrm{p} 1} / t_{\mathrm{p} 2}\right)$ because the direction of dimerization corresponds to the direction of $t_{p 1}$ (intradimer) and $t_{p 2}$ (interdimer).

Table II shows the following results. (i) In the case of $\beta$-(BEDT-TTF $)_{2} \mathrm{I}_{3}$, the lowpressure behavior of $T_{c}$ cannot be reproduced by the two-band model, but it can be reproduced by the dimer model. (ii) In the case of $\beta$-(BDA-TTP $)_{2} \mathrm{SbF}_{6}$, the low-pressure behavior of $T_{c}$ can be reproduced by both the dimer model and the two-band model. We also observe that the dimer model is more suitable than the two-band model, because the maximum of $T_{c}$ can be reproduced by the dimer model. (iii) In the case of $\beta$-(BDA$\mathrm{TTP})_{2} \mathrm{AsF}_{6}$, the low-pressure behavior of $T_{c}$ cannot be reproduced by the dimer model, but it can be reproduced by the two-band model. We also observe that the two-band model can reproduce the maximum of $T_{c}$. Thus, when the dimerization factor $\left(t_{\mathrm{p} 1} / t_{\mathrm{p} 2}\right)$ is large, which corresponds to the case of strong dimerization, experimental results are well-reproduced by the dimer model. On the other hand, when the dimerization factor is small, the results of the two-band model are suitable for reproducing the experimental results.

One may consider that the results of the dimer model are reproduced by the twoband model. However, we find that the results of the dimer model are not always the same as those of the two-band model with finite $U=10 t_{q 1}$ because the dimer model corresponds to the two-band model with $U \rightarrow \infty$. The dimer model has the effective $U_{\text {dimer }}=2 t_{p 1}$ at the limit of $U \rightarrow \infty$. In fact, we cannot find the value of $U$, using which the results of $\beta$-(BEDT-TTF $)_{2} \mathrm{I}_{3}$ in the dimer model are reproduced in the two-band 
model.

Our calculation cannot reproduce the experimental results at high pressures. Our assumption of the uniform displacements of molecules at high pressures may be incorrect. Taking account of the nonlinear distortion of the lattice against the uniaxial compression is one of the future problems. Thus, in this paper, our calculation should be compared with the experimental results obtained at low pressures.

As for the difference among the three materials, we observe that the calculated Fermi surfaces of $\beta$-(BDA-TTP $)_{2} \mathrm{SbF}_{6}$ and $\beta$-(BDA-TTP $)_{2} \mathrm{AsF}_{6}$ are slightly thinner than that of $\beta$-(BEDT-TTF $)_{2} \mathrm{I}_{3}$, which is consistent with the recent experimental results of magnetoresistance. ${ }^{33,34)}$

The structure of the spin susceptibility, which is very sensitive to the lattice structure, is important for the spin-fluctuation-mediated superconductivity. Thus, it may be essential that we employ the hopping integrals obtained by the first-principles calculation in order to discuss the effect of the uniaxial compression in more detail.

\section{Conclusions}

We have studied superconductivity under uniaxial compression in three $\beta$-type salts, $\beta$-(BEDT-TTF $)_{2} \mathrm{I}_{3}, \beta-(\mathrm{BDA}-\mathrm{TTP})_{2} \mathrm{SbF}_{6}$, and $\beta$-(BDA-TTP $)_{2} \mathrm{AsF}_{6}$, theoretically using both the two-band and dimer Hubbard models with FLEX approximation.

We have found that the behavior of $T_{c}$ in $\beta$-(BEDT-TTF $)_{2} \mathrm{I}_{3}$ with a stronger dimerization is reproduced by the dimer model, while that in weekly dimerized $\beta$-BDA-TTP salts is reproduced by the two-band model. The present study will serve to clarify whether the dimerization of molecules is a prerequisite for high $T_{\mathrm{C}}$ in organic superconductors.

Although we apply the uniaxial compression monotonically, the shift of $T_{c}$ exhibits a nonmonotonic behavior against the pressure. This result is theoretically considered to be due to the competition between the spin frustration and the effect of self-energy induced by the spin fluctuation.

\section{Acknowledgments}

This work was supported by a Grant-in-Aid from MEXT, Japan, for Scientific Research (Grant No.22540365) from the Ministry of Education, Culture, Sports, Science and Technology of Japan. Numerical calculations were performed at the Computer Center and the ISSP Supercomputer Center of the University of Tokyo, and at the Research 
Center for Computational Science, Okazaki, Japan. 


\section{References}

1) H. Urayama, H. Yamochi, G. Saito, K. Nozawa, T. Sugano , M. Kinoshita, S. Sato, K. Oshima, A. Kawamoto, and J. Tanaka: Chem. Lett. 17 (1988) 55.

2) H. Kino and H. Fukuyama: J. Phys. Soc. Jpn. 65 (1996) 2158.

3) H. Kino and H. Kontani: J. Phys. Soc. Jpn. 67 (1998) 3691.

4) H. Kondo and T. Moriya: J. Phys. Soc. Jpn. 67 (1998) 3695.

5) B. J. Powell and R. H. McKenzie: J. Phys.: Condens. Matter 18 (2006) R827.

6) K. Kuroki, T. Kimura, R. Arita, Y. Tanaka, and Y. Matsuda: Phys. Rev. B 65 (2002) 100516(R).

7) T. Watanabe, H. Yokoyama, Y. Tanaka, and J. Inoue: J. Phys. Soc. Jpn. 75 (2006) 074707.

8) T. Komatsu, N. Matsukawa, T. Inoue, and G. Saito: J. Phys. Soc. Jpn. 65 (1996) 1340 .

9) Y. Shimizu, K. Miyagawa, K. Kanoda, M. Maesato, and G. Saito: Phys. Rev. Lett. 91 (2003) 107001.

10) M. Maesato, Y. Kaga, R. Kondo, and S. Kagoshima: Rev. Sci. Instrum. 71 (2000) 176.

11) M. Maesato, Y. Shimizu, T. Ishikawa, and G. Saito: Synth. Met. 137 (2003) 1243.

12) H. Ito, T. Ishihara, H. Tanaka, S. I. Kuroda, T. Suzuki, S. Onari, Y. Tanaka, J. I. Yamada, and K. Kikuchi: Phys. Rev. B 78 (2008) 172506.

13) H. Ito, T. Ishihara, M. Niwa, T. Suzuki, S. Onari, Y. Tanaka, J. Yamada, H. Yamochi, and G. Saito: Physica B 405 (2010) S262.

14) N. E. Bickers, D. J. Scalapino, and S. R. White: Phys. Rev. Lett. 62 (1989) 961.

15) N. E. Bickers and D. J. Scalapino: Ann. Phys. (N.Y.) 193 (1989) 206.

16) K. Nomura, R. Muraoka, N. Matsunaga, K. Ichimura, and J. Yamada: Physica B $404(2009) 562$.

17) Y. Shimojo: private communication.

18) K. Ichimura, M. Takami, and K. Nomura: J Phys. Soc. Jpn. 77 (2008) 114707; K. Ichimura and K. Nomura: J. Phys. Soc. Jpn. 75 (2006) 051012.

19) Y. Tanaka and S. Kashiwaya: Phys. Rev. Lett. 74 (1995) 3451; S. Kashiwaya and Y. Tanaka: Rep. Prog. Phys. 63 (2000) 1641. The zero bias conductance peak in 
scanning tunneling spectroscopy corresponds to the presence of the Andreev bound state specific to anisotropic pairing, where gap function changes sign on the Fermi surface.

20) K. Nomura, N. Shirai, N. Matsunaga, K. Ichimura, and J. Yamada: Abstract Book in ICSM 2010.

21) T. Suzuki, S. Onari, H. Ito, and Y. Tanaka: Physica C 469 (2009) 979.

22) H. Kontani: Phys. Rev. B 67 (2003) 180503(R).

23) T. Mori, A. Kobayashi, Y. Sasaki, H. Kobayashi, G. Saito, and H. Inokuchi: Bull. Chem. Soc. Jpn. 57 (1984) 627.

24) W. Kang, G. Montambaux, J. R. Cooper, D. Jerome, P. Batail, and C. Lenoir: Phys. Rev. Lett. 62 (1989) 2559.

25) D. Beckmann, S. Wanka, J. Wosnitza, D. Schweitzer, and W. Strunz: Z. Phys. B 104 (1997) 207.

26) H. Tanino, K. kato, M. Tokumoto, H. Anzai, and G. Saito: J. Phys. Soc. Jpn. 54 (1985) 2390 .

27) S. Koikegami, S. Fujimoto, and K. Yamada: J. Phys. Soc. Jpn. 66 (1997) 1438.

28) H. Kontani and K. Ueda: Phys. Rev. Lett. 80 (1998) 5619.

29) K. Murata, M. Tokumoto, H. Anzai, H. Bando, G. Saito, K. Kajimura, and T. Ishiguro: J. Phys. Soc. Jpn. 54 (1985) 1236.

30) T. Mori, A. Kobayashi, Y. Sakai, H. Kobayashi, G. Saito, and H. Inokuchi: Chem. Lett. 13 (1984) 957.

31) J. Yamada, M. Watanabe, H. Akutsu, S. Nakatsuji, H. Nishikawa, I. Ikemoto, and K. Kikuchi: J. Am. Chem. Soc. 123 (2001) 4174.

32) Y. Nonoayama, Y. Maekawa, A. Kobayashi, Y. Suzumura, and H. Ito: J. Phys. Soc. Jpn. 77 (2008) 094703.

33) E. S. Choi, E. Jobilong, A. Wade, E. Goetz, J. S. Brooks, J. Yamada, T. Mizutani, T. Kinoshita, and M. Tokumoto: Phys. Rev. B 67 (2003) 174511.

34) S. Yasuzuka, S. Uji, H. Satsukawa, M. Kimata, T. Terashima, H. Koga, Y. Yamamura, K. Saito, H. Akutsu, and J. Yamada: Physica B 405 (2010) S288. 